\title{
Ultrahigh energy cosmic rays and new particle physics $^{1}$
}

\author{
M. Kachelrieß \\ TH Division, CERN, CH-1211 Geneva 23
}

\begin{abstract}
The current status of the UltraHigh Energy Cosmic Ray (UHE CR) enigma and several proposed solutions involving particle physics beyond the standard model are discussed. Emphasis is given to top-down models, and as a main example, supermassive dark matter as galactic source for UHE CR and the status of its experimental signatures (galactic anisotropy, chemical composition and clustering) is reviewed. Then different approaches to calculate fragmentation spectra of supermassive particles are discussed. Finally, it is argued that UHE neutrinos cannot be - neither directly or indirectly - responsible for the observed vertical air showers.
\end{abstract}

\section{INTRODUCTION}

Cosmic Rays [1] (CR) are observed in a wide energy range, starting from subGeV energies up to $3 \times 10^{20} \mathrm{eV}$. Apart from the highest energies, these particles are accelerated in our Galaxy, most probably by shocks produced by SNII explosions. Since the galactic magnetic field cannot confine and isotropize particles with energy higher than $\sim Z \times 10^{19} \mathrm{eV}$, it is natural to think that the UltraHigh Energy (UHE) component has an extragalactic origin. Moreover, the acceleration of protons or nuclei up to $2-3 \times 10^{20} \mathrm{eV}$ is difficult to explain within the known astrophysical galactic sources.

The most prominent signature of extragalactic UHECR is the so called GreisenZatsepin-Kuzmin (GZK) cutoff [2]: the energy losses of protons sharply increase at $E_{\mathrm{GZK}} \approx 6 \times 10^{19} \mathrm{eV}$, owing to scattering on photons from the microwave background, $p+\gamma_{3 K} \rightarrow \Delta^{+} \rightarrow N+\pi$, reducing their mean free path length to less than $50 \mathrm{Mpc}$ or so. Nuclei exhibit a similar cutoff at the same energy, while photons have no sharp cutoff but an even shorter free mean path, because of pair-production on the radio background. Thus, the UHECR spectrum should dramatically steepen above $E_{\mathrm{GZK}}$ for any homogeneous distribution of proton or nuclei sources. However, the observed spectrum extends up to $3 \times 10^{20} \mathrm{eV}$ and this maximum seems to be caused by the limited exposure. Both spectra, the expected and the observed one, are shown in Fig. 1, taken from Ref. [3].

1) Invited talk at "CAPP 2000", Verbier, July 2000

CERN-TH 2000-324 


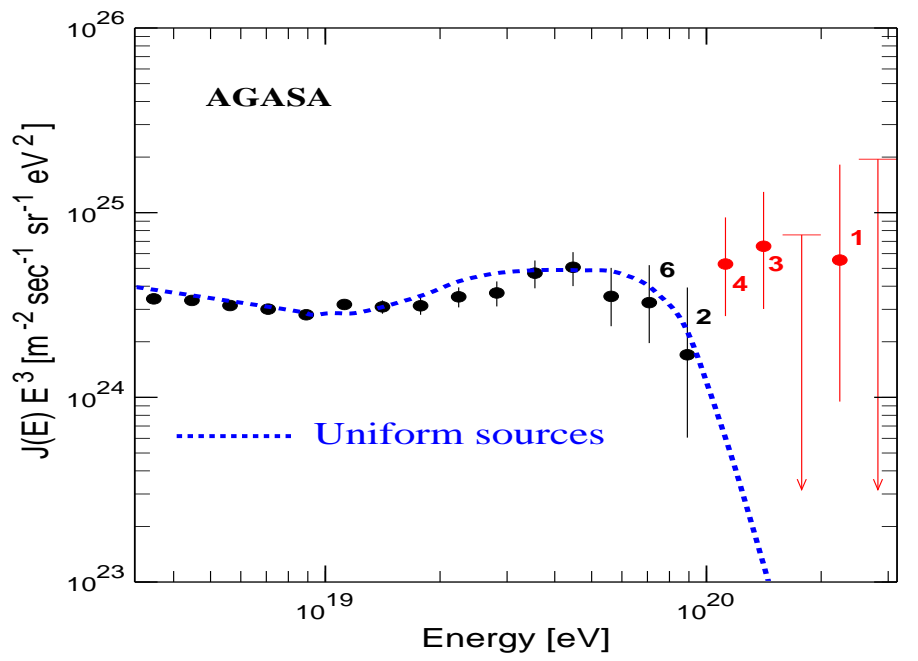

FIGURE 1. Energy spectrum as observed by AGASA. Error bars are $68 \%$ C.L., numbers are the number of events per energy bin. The dashed line is the spectrum expected from uniformly distributed astrophysical sources (from Ref. [3]).

There is another argument that disfavours the standard astrophysical sources: At energies $E \sim 10^{20} \mathrm{eV}$, the arrival direction of the primaries which is known within several degrees should point towards their site of origin. But no source of UHECR such us active galactic nuclei (AGN) has been found within $50 \mathrm{Mpc}$ in the direction of these events. In Fig. 2, the arrival directions of 92 events above $4 \times 10^{19} \mathrm{eV}$ are shown in galactic coordinates [4]. The events are scattered isotropically on larger scales, and no significant enhancement towards the galactic or supergalactic plane is found. Intriguingly, about $20 \%$ of the events are clustered in angular doublets or even triplets; both triplets are found near the supergalactic plane.

An elegant solution to some of the problems described above are top-down models: in contrast to the standard sources, the primaries are not accelerated but are the fragmentation products of some decaying superheavy particle $X$. For $X$ particles with mass $m_{X} \gtrsim 10^{12} \mathrm{GeV}$, the acceleration problem is solved trivially. Moreover, these sources also evade detection by normal astronomical methods.

This review is organised as follows: In Sec. II top-down models and in Sec. III their signatures are presented. We then discuss how the spectrum of hadrons produced in the decay of supermassive particles can be calculated. Finally, we argue in Sec. 5 that UHE neutrinos cannot be - neither directly or indirectly via resonant annihilations on relic neutrinos - responsible for the observed UHECR events.

For a review of astrophysical sources, we refer the interested reader to Ref. [5]. The suggestion that the violation of Lorentz invariance induced by quantum gravity effects avoids the GZK cutoff is covered in the recent review [6]. 


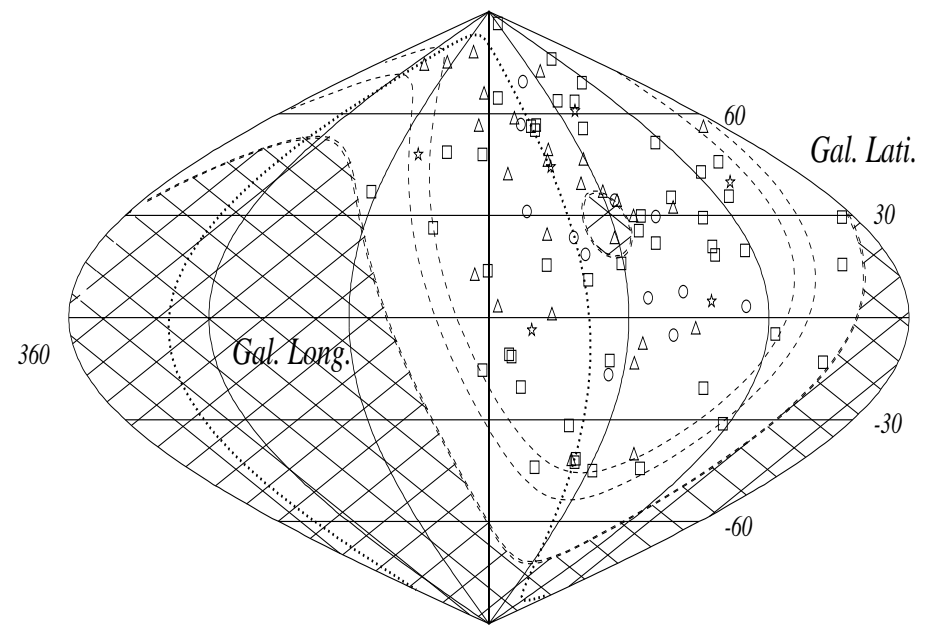

FIGURE 2. Map of 92 events above $4 \times 10^{19} \mathrm{eV}$ in galactic coordinates (from Ref. [4]). The supergalactic plane is shown by the dotted line, the hatched region is unobservable.

\section{TOP-DOWN MODELS}

Top-down model is a generic name for all proposals in which the observed UHECR primaries are produced as decay products of some superheavy particles $X$. These $X$ particles can be either metastable or be emitted by topological defects at the present epoch.

1. Topological defects (TD) [7] such as (superconducting) cosmic strings, monopoles, and hybrid defects can be effectively produced in non-thermal phase transitions during the preheating stage [8]. Therefore the presence of TDs is not in conflict with an inflationary period of the early Universe. They can naturally produce particles with large enough energies but fail generally to produce the observed flux of UHE primaries: The observation of the low energy diffuse gamma radiation by EGRET limits the energy dumped by high-energy particles into electromagnetic cascades and thereby also severely the possible UHECR flux. Another general reason for the low fluxes is the large distance between TDs, which is often comparable to the Hubble radius. Then the flux of UHE particles is either exponentially suppressed or strongly anisotropic if a TD is by chance nearby.

Ordinary strings can produce UHE particles e.g. when string loops self-intersect or when two cusp segments overlap and annihilate. In the latter case, the maximal energy of the produced fragmentation products is not $m_{X} / 2$ but can be much larger due to the high Lorentz factors of the ejected $X$ particles.

Superconducting strings: Cosmic strings can be superconducting in a broad class of particle models. Electric currents can be induced in the string either by a primordial magnetic field that decreases during the expansion of the Universe or when the string moves through galactic fields at present. If the Fermi momentum 
of the the trapped particles exceeds their mass outside the string, they can leave the string and decay.

Monopolium $M$ - boundstates of monopole-antimonopole pairs - was the first TD proposed as UHECR source [9]. It clusters like Cold Dark Matter (CDM) and is therefore an example of a SuperHeavy Dark Matter (SHDM) particle. The galactic density of monopoles is constrained by the Parker limit: the galactic magnetic field should not be eliminated by the acceleration of monopoles. Reference [10] concluded that the resulting limit on the UHECR flux produced by Monopolium annihilation is 10 orders of magitude too low.

Cosmic necklaces are hybrid defects consisting of monopoles connected by a string. These defects are produced by the symmetry breaking $G \rightarrow H \times U(1) \rightarrow$ $H \times Z_{2}$, where $G$ is semi-simple. In the first phase transition at scale $\eta_{m}$, monopoles are produced. At the second phase transition, at scale $\eta_{s}<\eta_{m}$, each monopole gets attached to two strings. Strings lose their energy and can contract through gravitational radiation. As a result, all monopoles annihilate in the end producing $X$-particles. Reference [11] argued that for a reasonable range of parameters the model predicts a UHECR flux close to the observed one. Recently, a numerical study [12] of the evolution of necklaces found that the lifetime of necklaces is generally much shorter than the age of the Universe $t_{0}$. A possible exception is the case $\eta_{m} \gg \eta_{s} \sim 100 \mathrm{GeV}[10]$, not a very attractive possibility in view of the limits on the $Z^{\prime}$ boson mass, $m_{Z^{\prime}} \gtrsim 600 \mathrm{GeV}$.

2. Superheavy metastable relic particles (SHDM) were proposed in Refs. [13,14] as UHECR source. They constitute (part of) the CDM and, consequently, their abundance in the galactic halo is enhanced by a factor $\sim 5 \times 10^{4}$ above their extragalactic abundance. Therefore, the proton and photon flux is dominated by the halo component and the GZK-cutoff is avoided, as was pointed out in Ref. [13]. The quotient $r_{X}=\Omega_{X}\left(t_{0} / \tau_{X}\right)$ of relic abundance $\Omega_{X}$ and lifetime $\tau_{X}$ of the $X$ particle is fixed by the UHECR flux, $r_{X} \sim \mathcal{O}\left(10^{-11}\right)$.

The relative abundance of particles that were in thermal equilibrium and then freeze-out is $\Omega h^{2} \propto 1 /\left(\sigma_{\text {ann }} v\right)$. Assuming for the annihilation cross-section $\sigma_{\text {ann }} \lesssim$ $1 / m_{X}^{2}$ one obtains the bound $m_{X} \lesssim 1 \mathrm{TeV}$. Therefore SHDM particles should never have been in thermal equilibrium and the challenge is to create create only few of them in a natural way.

There exist several plausible non-equilibrium production mechanisms. The most promising one is the gravitational production of the $X$ particles by the nonadiabatic change of the scale factor at the end of inflation [15]. In this scenario, the gravitational coupling of the $X$-field to the background metric yields the present abundance $\Omega_{0} \sim 1$ for $M_{X} \sim 10^{13} \mathrm{GeV}$, independent of any specific particle physics model. Other mechanisms proposed are thermal production during reheating, production through inflaton decay at the preheating phase, or through the decay of hybrid defects.

The lifetime of the superheavy particle has to be in the range $10^{17} \mathrm{~s} \lesssim \tau_{X} \lesssim 10^{28} \mathrm{~s}$, i.e. longer or much longer than the age of the Universe. Therefore it is an obvious 
question to ask if such an extremely small decay rate can be obtained without fine-tuning. A well-known example of how metastability can be achieved is the proton: in the standard model B-L is a conserved global symmetry, and the proton can decay only via non-renormalizable operators. Similarly, the $X$-particle could be protected by a new global symmetry which is only broken by higher-dimension operators suppressed by $M^{d}$, where for instance $M \sim M_{\mathrm{Pl}}$ and $d \geq 7$ is possible. The case of discrete gauged symmetries has been studied in detail in Refs. [16]. Another possibility is that the global symmetry is broken only non-perturbatively, either by wormhole [13] or instanton [14] effects. Then an exponential suppression of the decay process is expected and lifetimes $\tau_{X} \gg t_{0}$ can be naturally achieved.

An example of a SHDM particle in a realistic particle physics model is the crypton [17]. Cryptons are boundstates from a strongly interacting hidden sector of string/M theory. Their mass is determined by the non-perturbative dynamics of this sector. For example, flipped SU(5) motivated by string theory contains boundstates with mass $\sim 10^{12} \mathrm{GeV}$ and $\tau \sim 10^{15} \mathrm{yr}[18]$.

\section{THE SIGNATURES OF TOP-DOWN MODELS}

Superheavy dark matter has three clear signatures: 1. No GZK-cutoff, instead a flat spectrum (compared to astrophysical sources) up to $m_{X} / 2$. 2. High neutrino and photon fluxes compared to the proton flux. 3. Galactic anisotropy. Possibly, the observed small-scale clustering gives additional constraints.

Chemical composition: Since at the end of the QCD cascade quarks combine more easily to mesons than to baryons, the main component of the UHE flux are neutrinos and photons from pion decay with only a small admixture $(\sim 5 \%)$ of nucleons (cf. also Fig. 3). The differentiation between photon- and proton-induced air showers is however rather difficult at the highest energies: the muon content of photoninduced showers becomes at UHE similar to that of proton-induced showers [19]. The Landau-Pomeranchuk-Migdal effect reduces the electromagnetic interactions at these energies, while the geomagnetic field makes the processes $\gamma \rightarrow e^{+} e^{-}$and $\gamma \rightarrow \gamma \gamma$ possible. Reference [20] finds that above $4 \times 10^{19} \mathrm{eV}$ less than $55 \%$ of the UHE primaries can be photons. Since the AGASA data suggest that "normal" sources dominate the flux up to $10^{20} \mathrm{eV}$ and the flux is steeply falling with energy, this results seems to be still not problematic for SHDM models. Recently, Ref. [21] claimed that the Flye's Eye event at $3 \times 10^{20} \mathrm{eV}$ is inconsistent with a proton or photon as primary. They compared the event with the average depths from simulations instead of either only comparing the shape of the showers or taking into account the fluctuations of the first interaction point. Thus their conclusions, that do not agree with those of Ref. [22], seem to be artificially strong. Future fluorescence light detectors like HiRes [23] which are able to measure the complete shower development will reliably distinguish between photon and proton as primaries.

Galactic anisotropy: Dubovsky and Tinyakov noted that the UHECR flux from SHDM should show a galactic anisotropy, because the Sun is not in the centre of 
the Galaxy [25]. The degree of this anisotropy depends on how strong the CDM is concentrated near the galactic centre - a question currently under debate. Since the galactic center cannot be observed by present detectors, the predicted anisotropy is rather small. Reference [26] performed a harmonic analysis of the UHECR flux predicted by the SHDM model and found reasonable agreement with the AGASA data above $4 \times 10^{19} \mathrm{eV}$, although the statistical significance of these results is small. Similar results were obtained in Ref. [27]. We thus have to wait for the AUGER detector [24] which is currently built in Argentina and can see the galactic centre for a definite answer.

Clustering: Although the distribution of UHECR arrival directions is consistent with isotropy on larger scales, it shows an enhanced rate of clustering: The set of 92 UHECR with $E \geq 4 \times 10^{19} \mathrm{eV}$ contains 2 triplets and 7 doublets; above $E \geq 10^{20} \mathrm{eV}$, there are 2 doublets within 14 UHECR [4]. The chance probability to observe the clustered events in the case of an isotropic distribution of arrival directions was estimated to be $<1 \%$.

Waxman, Fisher and Piran pointed out [28] that the number density $n$ of uniformly distributed UHECR sources can be strongly constrained by the fraction of clustered events. As $n$ decreases, the sources have to become brighter for a fixed UHECR flux and therefore the probability for clustering increases. The analysis of Ref. [29] showed that $\sim 400$ sources should be inside the GZK volume, compared to $\sim 10$ GRB sources or $\sim 250$ AGNs of which only a small fraction is thought to be UHECR sources. However, the statistical uncertainties are still very large.

The clustering probability was discussed for SHDM in Ref. [30]. The authors assumed a smooth NFW profile $\left(n(r) \propto r^{-1}\right.$ for $\left.r \rightarrow 0\right)$ for the SHDM plus a clumped component and obtained a rather good agreement with the observed clustering statistics. Table 1 shows the experimentally observed number of doublets $N_{2}$ within $3^{\circ}, 4^{\circ}$ and $5^{\circ}$ compared with the average numbers $\left\langle N_{2}\right\rangle$ obtained in the simulation. Also shown is the probability $p$ to observe $N_{2}$ or more doublets. Without

TABLE 1.

\begin{tabular}{ll|c|c|c}
\hline & $3^{\circ}$ & $4^{\circ}$ & $5^{\circ}$ & \\
$N_{2}$ & 12 & 14 & 20 & \\
$\left\langle N_{2}\right\rangle$ & 8 & 14 & 21 & \\
$p\left(\left\langle N_{2}\right\rangle \geq N_{2}\right)$ & $12 \%$ & $47 \%$ & $57 \%$ \\
\hline
\end{tabular}

the clumped component, the number of doublets drops typically by 1-2, i.e. is still higher than expected from an isotropic distribution.

The signatures of TD models are not so clear. The high photon/proton ratio at generation can be masked by the strong absorption of UHE photons, but it is still higher than expected from astrophysical sources. A crucial test can be the (non-) observation of the large flux of UHE neutrinos predicted in all top-down models. Finally, the GZK-cutoff is less pronounced for TDs than for astrophysical sources, because of the flatter generation spectrum of the UHE particles. 


\section{FRAGMENTATION SPECTRUM OF HADRONS}

To make detailed predictions about the energy spectrum of UHECR in top-down models, the spectrum of hadrons produced in QCD cascades has to be known. Since $m_{X}$ is much above the supposed mass scale of supersymmetric particles, SUSY partons (gluinos and squarks) should be included in the cascade development. There are different strategies to do this:

- Analytical solutions of the DGLAP equations, in particular the limiting spectrum, are known to describe quite accurately the observed hadron spectra produced in $e^{+} e^{-}$annihilations. Therefore the limiting spectrum for SUSYQCD was derived and proposed as a useful description of hadron spectra at large $m_{X}$ in Ref. [31].

However, two assumptions are used in the derivation of the limiting spectrum which prevent it from being useful in the case of SUSY-QCD: First, the number of active flavours $n_{f}$ has to be kept constant. Second, the cutoff $Q_{0}$ for the perturbative evolutions of the QCD cascade is identified with $\Lambda$, which fixes the strength of the strong coupling via $\alpha_{s}\left(Q^{2}\right)=4 \pi / b \ln \left(Q^{2} / \Lambda^{2}\right)$. This identification makes sense for $n_{f}=3$, when both parameters are $\sim 250 \mathrm{MeV}$, but not for $n_{f}=6$ or even above the SUSY threshold.

- Another possibility is to evolve the fragmentation functions $D_{i}^{a}$ measured at low energies to the higher scale $m_{X}$ with the DGLAP equations [32],

$$
\frac{\partial}{\partial \ln Q^{2}} D_{i}^{a}\left(x, Q^{2}\right)=\sum_{j} \int_{x}^{1} \frac{d z}{z} \frac{\alpha_{s}\left(Q^{2}\right)}{2 \pi} P_{j i}(z) D_{j}^{a}\left(x / z, Q^{2}\right) .
$$

In this approach, the region of accessible $x$ values is restricted by the lowenergy data.

- Available Monte Carlo simulations such as HERWIG or JETSET have precision, time and memory problems for large $m_{X}$ and can be used, if at all, only after modifications. Furthermore, supersymmetric partons are only included as resonances at present, but not in the QCD cascade. In Ref. [33], HERWIG was used to calculate the spectra of stable particles produced in $X$ decay within QCD. It was found that the proton flux at $x \gtrsim 0.2$ can compete with the photon and neutrino flux. However, it was realized later that the overproduction of baryons at large $x$ is an artifact of the hadronization procedure used in HERWIG [32].

In Ref. [34], the results of a new Monte Carlo simulation, especially written for UHE, were presented. The simulation includes SUSY partons and a resulting spectrum for UHECR is shown in Fig. 3. An advantage of this approach is that also the spectrum of the Lightest Supersymmetric Particle (LSP) can be calculated, which is stable if $R$-parity is conserved. The energy fraction taken away by the LSP can be considerable [35] owing to its hard spectrum and was found to be $\sim 10 \%$. 


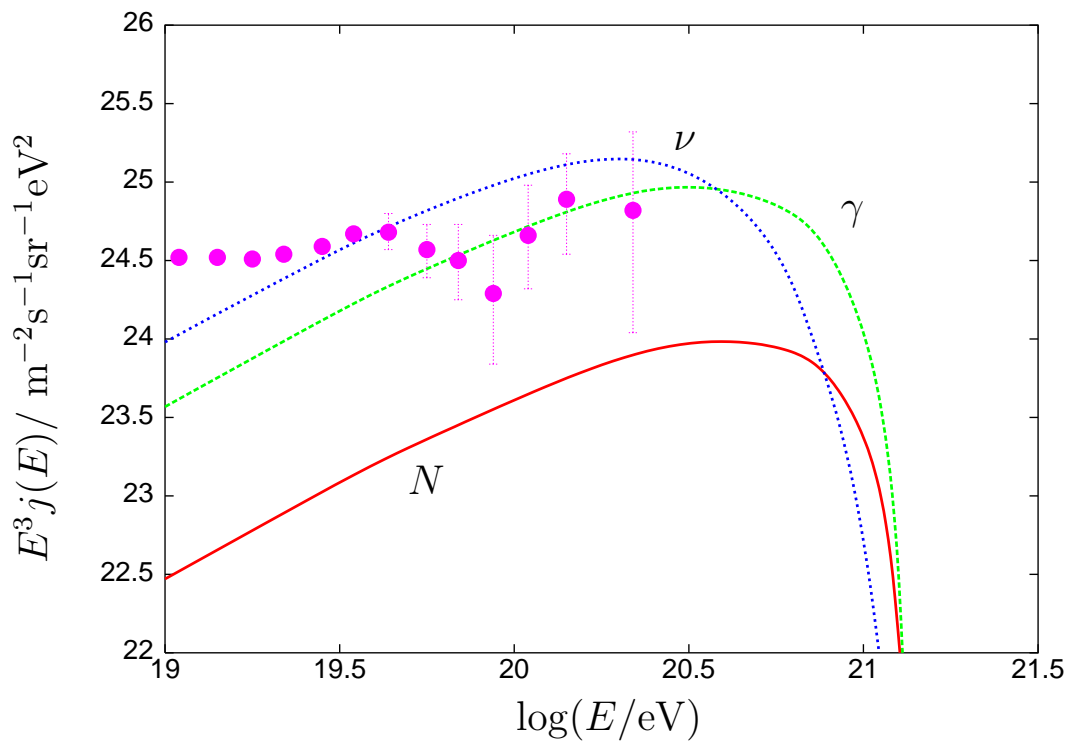

FIGURE 3. Flux of UHE particles from $X$-particle decay with $m_{X}=3 \times 10^{12} \mathrm{GeV}$. The fragmentation spectra are from the Monte Carlo simulation [34]; only the halo component is shown.

\section{UHE NEUTRINOS}

Neutrinos are the only known stable particles that can traverse extragalactic space without attenuation even at energies $E \gtrsim E_{\mathrm{GZK}}$, thus avoiding the GZK cutoff. Therefore, it has been speculated that the UHE primaries initiating the observed air showers are not protons, nuclei or photons but neutrinos [36-38]. However, neutrinos are in the Standard Model (SM) deeply penetrating particles producing only horizontal not vertical Extensive Air Showers (EAS). Therefore, either one has to postulate new interaction that enhance the UHE neutrino-nucleon cross-section or the neutrino has to be converted locally into a strongly interacting hadron.

\section{A Annihilations on relic neutrinos}

In the later scheme [39], UHE neutrinos from distant sources annihilate with relic neutrinos on the $Z$ resonance. The fragmentation products from nearby $Z$ decays are supposed to be the primaries responsible for the EAS above the GZK-cutoff. For energies of the primary neutrino of $E_{\nu} \sim 10^{23} \mathrm{eV}$, the mass of the relic neutrino should be $m_{\nu}=m_{Z}^{2} /\left(2 E_{\nu}\right) \sim 0.1 \mathrm{eV}$ which is compatible with atmospheric neutrino data. There are, however, severe observational constraints on this model:

1. Since the Pauli principle does not allow arbitrary densely packed neutrinos, an upper limit for their number density $n$ in the galactic halo is $n \leq(4 \pi / 3) p_{\max }^{3}$, where 
$p_{\max } \sim m_{\nu} v_{\text {rot }}$ and $v_{\text {rot }} \sim 220 \mathrm{~km} / \mathrm{s}$ is the Galactic rotation speed. A somewhat better limit comes from the requirement that during the gravitational collapse the neutrino phase space density does not increase. Therefore the overdensity of relic neutrinos is small and one expects in this model a rather pronounced GZK-cutoff.

2. Since the interaction probability for a UHE neutrino in the neutrino halo is small, a large neutrino flux is needed to produce the observed UHECR. The limit on horizontal EAS set by the Fly's Eye experiment [40] limits therefore severely this model: Ref. [41] found that the neutrino spectrum has to be extremely flat, $d N / d E \propto E^{-\gamma}$ with spectral index $\gamma<1.2$. Even if one assumes a large neutrino enhancement factor due to a lepton asymmetric Universe, the spectrum has to be much flater, $\gamma<1.8$, than expected from astrophysical sources.

3. The observed UHECR flux implies an upper bound on the UHE neutrino flux produced in astrophysical sources which are not hidden. If UHE neutrinos annihilating on relic neutrinos contribute significantly to the observed UHECR at $\sim 10^{20} \mathrm{eV}$, a new class of UHE neutrino source has to be invoked which is optically thick for nucleons. The energy generation of these sources was estimated to be comparable to the total photon luminosity of the Universe [42].

\section{B UHE neutrino and weak-scale string theories}

Most models introducing new physics at a scale $M$ to produce large cross-sections for UHE neutrinos fail because experiments generally constrain $M$ to be larger than the weak scale, $M \gtrsim m_{Z}$, and unitarity limits cross-sections to be $\mathcal{O}\left(\sigma_{\text {tot }}\right) \lesssim$ $1 / M^{2} \lesssim 1 / m_{Z}^{2}$. String theories with large extra dimensions [43] are different in this respect: if the SM particles are confined to the usual $3+1$-dimensional space and only gravity propagates in the higher-dimensional space, the compactification radius $R$ of the large extra dimensions can be large, corresponding to a small scale $1 / R$ of new physics. The weakness of gravitational interactions is a consequence of the large compactification radius, since Newton's constant is then given by $G_{N}^{-1}=$ $8 \pi R^{\delta} M_{D}^{\delta+2}$, where $\delta$ is the number of extra dimensions and $M_{D} \sim \mathrm{TeV}$ is the fundamental mass scale. Such a scenario is naturally realized in theories of open strings, where SM particles correspond to open strings beginning and ending on D-branes, whereas gravitons correspond to closed strings which can propagate in the higher-dimensional space. From a four-dimensional point of view the higher dimensional graviton in these theories appears as an infinite tower of Kaluza-Klein (KK) excitations with mass squared $m_{\vec{n}}^{2}=\vec{n}^{2} / R^{2}$. Since the weakness of the gravitational interaction is partially compensated by the large number of KK states and cross-sections of reactions mediated by spin 2 particles are increasing rapidly with energy, it has been argued in Refs. [37,38] that neutrinos could initiate the observed vertical showers at the highest energies.

In the calculations of Refs. [38,44] it was assumed that the massless fourdimensional graviton and its massive KK excitations couple with the usual gravitational strength $\bar{M}_{\mathrm{Pl}}^{-1}=\sqrt{8 \pi} / M_{\mathrm{Pl}}$. Then the sum over all KK contributions to a 




FIGURE 4. Neutrino-nucleon cross-section $\sigma_{\nu N} /$ mbarn due to $W$-exchange (CC) and exchange of KK gravitons as function of $\log \left(E_{\nu} / \mathrm{eV}\right)$ for $M_{\mathrm{st}}=6,15$ and $30 \mathrm{TeV}$. All for $\delta=2$ and $c=1$.

given ( $t$-channel) scattering amplitude,

$$
M_{f i} \propto P(t)=\sum_{\vec{n}=1}^{\infty} \frac{g_{\vec{n}}}{t-m_{\vec{n}}^{2}}, \quad \vec{n}=\left(n_{1}, \ldots, n_{\delta}\right)
$$

only converges in the case of one extra dimension, and for two or more extra dimensions a cutoff has to be introduced by hand. However, it has been pointed out [45] that due to brane fluctuations the effective coupling $g_{\vec{n}}$ of the level $\vec{n} \mathrm{KK}$ mode to four-dimensional fields is suppressed exponentially,

$$
g_{\vec{n}}=\frac{1}{\bar{M}_{\mathrm{Pl}}} \exp \left(-\frac{c m_{\vec{n}}^{2}}{M_{\mathrm{st}}^{2}}\right),
$$

where $c$ is a constant of order 1 or larger, which parametrises the effects of a finite brane tension [45], and $M_{\text {st }} \sim M_{D}$ is the string scale ${ }^{2}$. This exponential suppression thereby provides a dynamical cutoff in the sum over all KK modes.

In Fig. 4, the cross-sections obtained in Ref. [47] due to KK exchange are shown for three different values of $M_{\mathrm{st}}$ together with the charged-current cross-section of the SM. It is clear that even for $\sigma_{N \nu}^{\mathrm{KK}}=10$ mbarn a value of $M_{\text {st }}$ not much above $1 \mathrm{TeV}$ is required. While present collider experiments do not exclude this possibility, SN 1987A gives $M_{D} \gtrsim 50 \mathrm{TeV}$ [48]. The latter limit was obtained for a rather conservative choice of supernova parameters, and therefore $M_{D} \lesssim 10 \mathrm{TeV}$

2) A similar suppression of the coupling to higher KK modes was found also in Ref. [46]. 


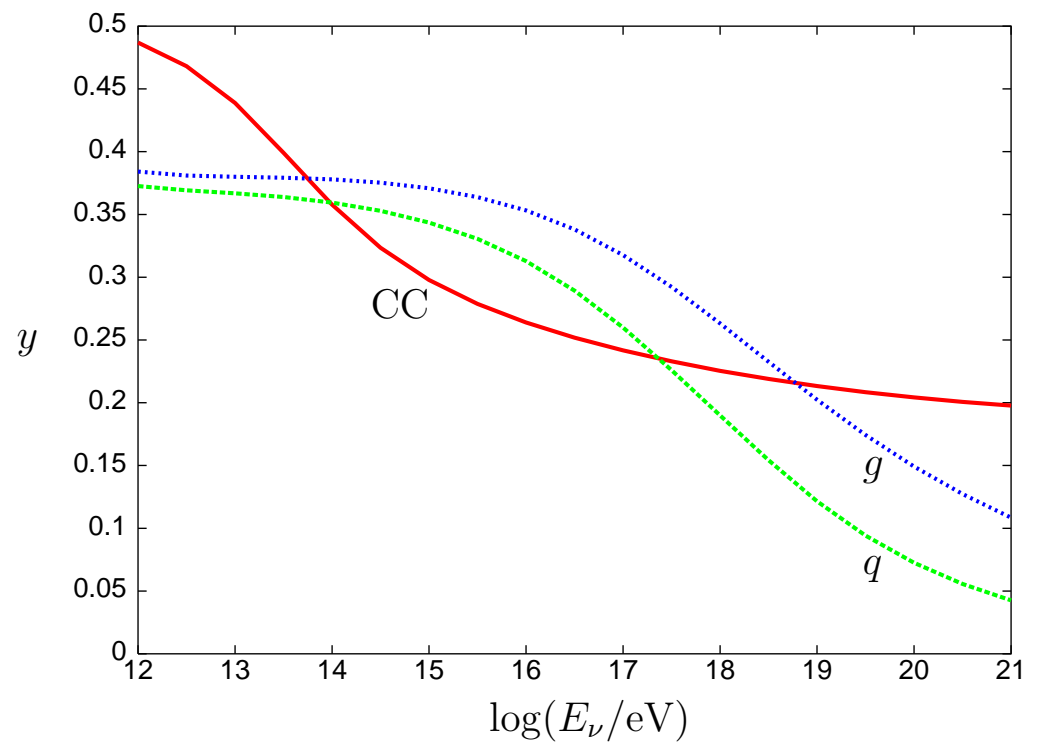

FIGURE 5. Energy transfer $y$ in the subreactions with $W$-exchange (CC), exchange of KK gravitons with quarks $(q)$ and gluons $(g)$ as function of $\log \left(E_{\nu} / \mathrm{eV}\right)$ for $M_{\mathrm{st}}=6 \mathrm{TeV}, \delta=2$ and $c=1$.

seems to be incompatible with SN 1987A even allowing for rather large astrophysical uncertainties.

The second important quantity characterising the development of an air shower besides $\sigma_{\text {tot }}$ is the energy transfer $y=\left(E_{\nu}-E_{\nu}^{\prime}\right) / E_{\nu}$. In contrast to charged-current scattering where the electromagnetic shower initiated by the charged lepton is practically indistinguishable from a hadronic shower, only the hit nucleon can initiate an air shower in KK scattering. Therefore, even a neutrino with large $\sigma_{\text {tot }}$ will behave like a penetrating particle if it does not transfer a large fraction of its energy per interaction to the shower. In Fig. 5, the energy transfer $y$ is shown as function of $E_{\nu}$. At energies of interest, $E_{\nu} \approx 10^{20} \mathrm{eV}$, the transferred energy fraction is only around $y \approx 0.1$, i.e., much smaller than $y \approx 0.6$ typical of nucleon-nucleon collisions.

Let us now discuss in a very general way how large the total cross-section of a UHE primary able to produce the observed vertical air showers should be. The survival probability $N$ at atmospheric depth $X$ of a primary with mean free path $\lambda=m_{\text {air }} / \sigma_{\text {tot }}$ is $N(X)=\exp (-X / \lambda)$, where $m_{\text {air }} \approx 2.4 \cdot 10^{-24} \mathrm{~g}$ is the weight of an "average" air atom. Hence, the probability distribution $p$ of the first interaction point $X_{1}$ has its maximum at $p\left(X_{1}\right)=\lambda$.

For a proton with energy $E=10^{20} \mathrm{eV}$, the mean free path is $\lambda_{p} \approx 40 \mathrm{~g} / \mathrm{cm}^{2}$ and thus a proton air shower is indeed initiated in the top of the atmosphere. After the first interaction, the number of particles in the shower grows until it reaches its maximum at $X_{\max } \approx 800 \mathrm{~g} / \mathrm{cm}^{2}$. Hence, a vertical proton air shower needs almost 
the complete atmosphere for its development.

How would this picture change for a neutrino with $\lambda_{\nu}=10 \lambda_{p}$, i.e., $\sigma_{\text {tot }}=$ 15 mbarn? Taking into account only the delayed start of the shower shifts the shower maximum already $\approx 360 \mathrm{~g} / \mathrm{cm}^{2}$ downwards in the atmosphere. The small energy fraction transferred to the shower by each interaction delays the shower development even further. Additionally, the fluctuations of a neutrino shower are enhanced compared to a proton shower. Hence, the shower evolution is clearly different from a proton shower. Therefore even neutrino-nucleon cross-sections as large as 15 mbarn due to KK exchange are not sufficient to explain vertical air showers by neutrino primaries.

Finally, we want to discuss the high-energy behaviour of the total cross-section $\sigma_{\text {tot }}$. A partial-wave analysis shows that at $\sqrt{s}=\mathcal{O}\left(M_{\text {st }}\right)$ the amplitude of $\nu+q \rightarrow$ $\nu+q$ starts to violate unitarity. At the same energy scale, one expects that the effective theory used to derive $\sigma_{N \nu}^{\mathrm{KK}}$ breaks down and that the growth of $\sigma_{N \nu}^{K K}$ is slowed down. In this case, the results shown in Fig. 4 would be an upper bound for $\sigma_{N \nu}^{\mathrm{KK}}$. Since a calculation of $\sigma_{N \nu}^{\mathrm{KK}}$ valid for $s \gg M_{\mathrm{st}}^{2}$ within string theory seems at present not to be feasible, it is interesting to ask if general principles uch as unitarity can be used at all as guidelines.

Khuri considered potential scattering on $\mathbb{R}^{3} \otimes S^{1}$ as a toy model for string theory with one large extra dimension in Ref. [49]. He showed that analytical properties of the forward scattering amplitude $T_{n n}(s, t=0)$, which are true in $\mathbb{R}^{3}$, do not necessarily hold in $\mathbb{R}^{3} \otimes S^{1}$ for $n>0$. At least in this specific example however, the forward scattering amplitude for $n=0$ ("SM particles") has the usual analytical properties known from $\mathbb{R}^{3}$. If this would hold true generally, then the Regge approach together with the eikonal method to ensure unitarity should give an idea of the high-energy behaviour of $\sigma_{\text {tot }}$. A general Regge amplitude $A_{R}$ can be represented by

$$
A_{R}(s, t)=\beta(t) s^{\alpha(t)},
$$

where the exponent $\alpha(t)$ is given by the relation between spin $\sigma_{i}=\operatorname{int}[\alpha(t)]$ and mass $m_{i}^{2}=t$ of the particles lying on the leading Regge trajectory contributing to the reaction. In our case, the intercept $\alpha(0)$ of this trajectory is equal to the spin of the massless graviton, $\alpha(0)=2$. String theory suggests that the Regge trajectories are linear, $\alpha(t)=\alpha_{0}+\alpha^{\prime} t$, and that their slope is given by the string tension, $\alpha^{\prime}=1 /\left(4 \pi M_{\mathrm{st}}^{2}\right)$. The residue

$$
\beta(t)=-\exp (-\mathrm{i} \alpha(t) \pi / 2) \mathrm{e}^{a t}
$$

contains the phase of the amplitude and the Reggeon coupling $\propto \exp (a t)$, for which Eq. (3) gives $a=c / M_{\text {st }}^{2}$. In $d=4$ dimensions, the energy dependence of the total cross-section follows as $\sigma_{\text {tot }}(s) \propto \ln ^{2}(s)$ [47]. The results of Ref. [50] suggest that for $s \gg M_{\mathrm{st}}^{2}$ and $\delta$ extra dimensions, $\ln ^{2}\left(s / s_{0}\right)$ should be replaced by $\ln ^{2+\delta}\left(s / s_{0}\right)$. 


\section{CONCLUSIONS}

Superheavy, metastable relic particles is the most promising source in the framework of top-down models. Its cleanest signature is the galactic anisotropy which should be easily detectable by a detector in the southern hemisphere as AUGER. Experimentally more difficult signatures are the high photon/proton ratio and the detection of the predicted large UHE neutrino fluxes via horizontal EAS. All topdown models predict also an appreciable LSP flux if R-parity is conserved.

Also in theories with large extra dimensions, neutrinos behave as deeply penetrating particles and are therefore not responsible for the observed UHECR events.

\section{ACKNOWLEDGEMENTS}

I am grateful to V. Berezinsky, S. Ostapchenko and M. Plümacher for very pleasant collaborations and useful discussions.

\section{REFERENCES}

1. For recent reviews of UHECR physics see V. Berezinsky, Nucl. Phys. (Proc. Suppl.) 70, 419 (1999); P. Bhattacharjee and G. Sigl, Phys. Rep. 327, 109 (2000); M. Nagano and A.A. Watson, Rev. Mod. Phys. 72, 689 (2000). The general field of CR physics is covered in V.L. Ginzburg (ed.), Astrophysics of Cosmic Rays, North-Holland, Amsterdam 1990.

2. K. Greisen, Phys. Rev. Lett. 16, 748 (1966); G.T. Zatsepin and V.A. Kuzmin, JETP Lett. 4, 78 (1966).

3. N. Hayashida et al., Appendix for Astrophys. J. 522, 225 (1999), astro-ph/0008102.

4. Y. Uchihori et al., Astropart. Phys. 13, 151 (2000).

5. C.P. Norman, D.B. Melrose and A. Achtenberg, Astrophys. J. 454, 60 (1995); R.D. Blandford, Phys. Scripta T85, 191 (2000).

6. J. Ellis, hep-ph/0010474.

7. A. Vilenkin and E.P.S. Shellard, Cosmic Strings and other Topological Defects, Cambrige University Press, 1994; M.B. Hindmarsh and T.W.B. Kibble, Rep. Prog. Phys. 58, 477 (1995).

8. S. Khlebnikov, L. Kofman, A. Linde and I. Tkachev, Phys. Rev. Lett. 81, 2012 (1998); V.A. Kuzmin and I.I. Tkachev, Phys. Rep. 320, 199 (1999).

9. C.T. Hill, Nucl. Phys. B224, 469 (1983).

10. J.J. Blanco-Pillado and K.D. Olum, Phys. Rev. D60, 083001 (1999).

11. V. Berezinsky and A. Vilenkin, Phys. Rev. Lett. 79, 5202 (1997).

12. X. Siemens, X. Martin and K. D. Olum, astro-ph/0005411.

13. V. Berezinsky, M. Kachelrieß and A. Vilenkin, Phys. Rev. Lett. 79, 4302 (1997).

14. V.A. Kuzmin and V.A. Rubakov, Phys. Atom. Nucl. 61, 1028 (1998).

15. D.J. Chung, E.W. Kolb and A. Riotto, Phys. Rev. D59, 023501 (1999); V. Kuzmin and I. Tkachev, JETP Lett. 68, 271 (1998)

16. K. Hamaguchi, Y. Nomura and T. Yanagida, Phys. Rev. D58, 103503 (1998); K. Hamaguchi, K. I. Izawa, Y. Nomura and T. Yanagida, Phys. Rev. D60, 125009 (1999). 
17. J. Ellis, J.L. Lopez and D.V. Nanopoulos, Phys. Lett. B247, 257 (1990).

18. K. Benakli, J. Ellis and D.V. Nanopoulos, Phys. Rev. D59, 047301 (1999).

19. F.A. Aharonian, B.L. Kanevsky and V.A. Sahakian, J. Phys. G17, 1909 (1991).

20. M. Ave et al., Phys. Rev. Lett. 85, 2244 (2000).

21. L.A. Anchordoqui et al., Phys. Lett. B492, 237 (2000).

22. N.N. Kalmykov, S.S. Ostapchenko and A.I. Pavlov, Nucl. Phys. (Proc. Suppl.) 52B, 17 (1997); G. Burdman, F. Halzen and R. Gandhi, Phys. Lett. B417, 107 (1998).

23. T. Abu-Zayyad et al., Nucl. Instrum. Meth. A450, 253 (2000).

24. D. Zavrtanik [AUGER Collaboration], Nucl. Phys. Proc. Suppl. 85, 324 (2000).

25. S.L. Dubovsky and P.G. Tinyakov, JETP Lett. 68, 107 (1998).

26. V. Berezinsky and A.A. Mikhailov, Phys. Lett. B449, 237 (1999); G.A. Medina Tanco and A.A. Watson, Astropart. Phys. 12, 25 (1999).

27. T. Wibig and A.W. Wolfendale, J. Phys. G26, 1033 (2000).

28. E. Waxman, K.B. Fisher and T. Piran, Astrophys. J. 483, 1 (1997).

29. S.L. Dubovsky, P.G. Tinyakov and I.I. Tkachev, Phys. Rev. Lett. 85, 1154 (2000).

30. P. Blasi and R. Sheth, Phys. Lett. B486, 233 (2000).

31. V. Berezinsky and M. Kachelrieß, Phys. Lett. B434, 61 (1998).

32. N.A. Rubin, Master of Philosophy Thesis, University of Cambridge, 1999.

33. M. Birkel and S. Sarkar, Astropart. Phys. 9, 297 (1998).

34. V. Berezinsky and M. Kachelrieß, hep-ph/0009053, to appear in Phys. Rev. D.

35. V. Berezinsky and M. Kachelrieß, Phys. Lett. B422, 163 (1998).

36. V.S. Berezinsky and G.T. Zatsepin, Phys. Lett. B28, 423 (1969); G. Domokos and S. Nussinov, Phys. Lett. B187, 372 (1987); J. Bordes, H. Chan, J. Faridani, J. Pfaudler and S.T. Tsou, Astropart. Phys. 8, 135 (1998).

37. G. Domokos and S. Kovesi-Domokos, Phys. Rev. Lett. 82, 1366 (1998).

38. P. Jain, D.W. McKay, S. Panda and J.P. Ralston, Phys. Lett. B484, 267 (2000).

39. D. Fargion, B. Mele and A. Salis, Astrophys. J. 517, 725 (1999); T.J. Weiler, Astropart. Phys. 11, 303 (1999).

40. R.M. Baltrusaitis et al., Phys. Rev. D31, 2192 (1985).

41. J.J. Blanco-Pillado, R.A. Vazquez and E. Zas, Phys. Rev. D61, 123003 (2000).

42. E. Waxman, astro-ph/9804028.

43. N. Arkani-Hamed, S. Dimopoulos and G. Dvali, Phys. Lett. B429, 263 (1998); I. Antoniadis, N. Arkani-Hamed, S. Dimopoulos and G. Dvali, Phys. Lett. B436, 257 (1998).

44. S. Nussinov and R. Shrock, Phys. Rev. D59, 105002 (1999).

45. M. Bando, T. Kugo, T. Noguchi and K. Yoshioka, Phys. Rev. Lett. 83, 3601 (1999); J. Hisano and N. Okada, Phys. Rev. D61, 106003 (2000).

46. L. Dixon, D. Friedan, E. Martinec and S. Shenker, Nucl. Phys. B282, 13 (1987); S. Hamidi and C. Vafa, Nucl. Phys. B279, 465 (1987); I. Antoniadis and K. Benakli, Phys. Lett. B326, 69 (1994).

47. M. Kachelrieß and M. Plümacher, Phys. Rev. D62, 103006 (2000).

48. For a review of experimental bounds, see e.g. M.E. Peskin, hep-ph/0002041.

49. N.N. Khuri, Ann. Phys. 242, 332 (1995).

50. M. Chaichian and J. Fischer, Nucl. Phys. B303, 557 (1988); V.A. Petrov, hep-ph/0008329. 